\title{
Bioreducible Hydrophobin-Stabilized Supraparticles for Selective Intracellular Release
}

\author{
Daniele Maiolo, ${ }^{\dagger, \|}$ Claudia Pigliacelli, ${ }^{\dagger, \perp, \| \odot ~ P a o l a ~ S a ́ n c h e z ~ M o r e n o, ~}{ }^{\dagger, \nabla}$ Martina Bruna Violatto, ${ }^{\ddagger}$ \\ Laura Talamini, ${ }^{\neq}$Ilaria Tirotta, ${ }^{\dagger}$ Rosanna Piccirillo, ${ }^{\ddagger}$ Massimo Zucchetti, ${ }^{*}$ Lavinia Morosi,

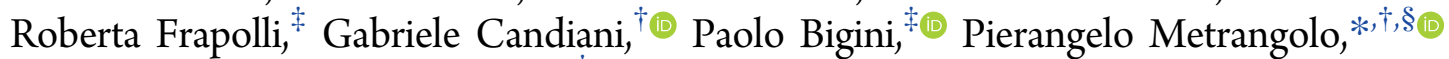 \\ and Francesca Baldelli Bombelli*, ${ }^{\dagger}$ (i)
}

\begin{abstract}
${ }^{\dagger}$ Interdepartmental Laboratory of Nanomedicine (NanoMedLab), Laboratory of Supramolecular and BioNano Materials (SupraBioNanoLab), and Fondazione Centro Europeo Nanomedicina (CEN), Department of Chemistry, Materials, and Chemical Engineering "Giulio Natta”, Politecnico di Milano, via L. Mancinelli 7, 20131 Milan, Italy

${ }^{\ddagger}$ IRCCS-Istituto di Ricerche Farmacologiche "Mario Negri”, 20156 Milano, Italy

${ }^{\S}$ VTT-Technical Research Centre of Finland Ltd, Biologinkuja 7, FI-02044 Espoo, Finland
\end{abstract}

\section{Supporting Information}

\begin{abstract}
One of the main hurdles in nanomedicine is the low stability of drug-nanocarrier complexes as well as the drug delivery efficiency in the region-of-interest. Here, we describe the use of the film-forming protein hydrophobin HFBII to organize dodecanethiol-protected gold nanoparticles (NPs) into well-defined supraparticles (SPs). The obtained SPs are exceptionally stable in vivo and efficiently encapsulate hydrophobic drug molecules. The HFBII film prevents massive release of the encapsulated drug, which, instead, is activated by selective SP disassembly triggered intracellularly by glutathione reduction of the protein film. As a consequence, the therapeutic efficiency of an encapsulated anticancer drug is highly enhanced ( 2 orders of magnitude decrease in $\mathrm{IC}_{50}$ ). Biodistribution and pharmacokinetics studies demonstrate the high stability of the loaded SPs in the bloodstream and the selective release of the payloads once taken up in the tissues. Overall, our results provide a rationale for the development of bioreducible and multifunctional nanomedicines.

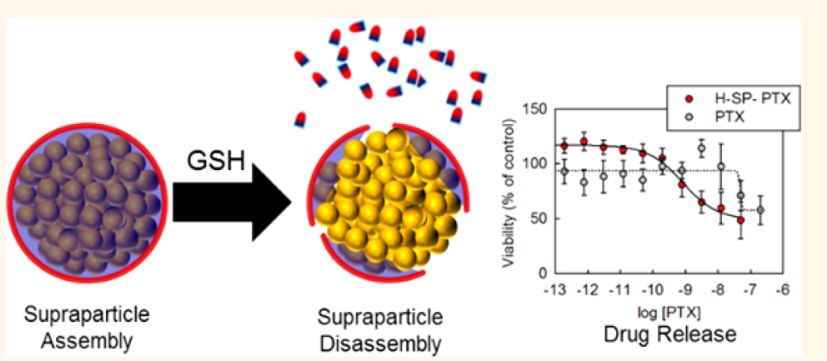

Supraparticle

Supraparticle Disassembly$$
\log [\text { PTX] }
$$

.
\end{abstract}

KEYWORDS: gold nanoparticle, supraparticle, self-assembly, nanobio interface, hydrophobin, drug release

\begin{abstract}
I $\mathrm{n}$ the last decades the use of nanosized objects has been extensively exploited for improving in vivo drug delivery. ${ }^{1}$ In fact, encapsulation of hydrophobic drugs into nanocarriers has been proven to increase their stability and circulating time in the blood as well as reduce their unspecific toxicity and cell resistance. ${ }^{2}$ Toward reaching these goals, a myriad of nanotechnology-based drug delivery systems has been deployed, comprising, among others, liposomes, coreshell nanoparticles (NPs) of different nature, and nanotubes. ${ }^{1,3,4}$ However, nanocarrier-based delivery has not yet reached the hoped results in clinics. ${ }^{5}$ One of the main hurdles is the low stability of drug-nanocarrier complexes in the biological environment, which often results in an undesired drug leakage in the biological fluids, in particular in the bloodstream for intravenously injected formulations. This often leads to two main side-effects: on one side, the penetration of drugs in the brain, heart, or other vital organs, and, on the other
\end{abstract}


a

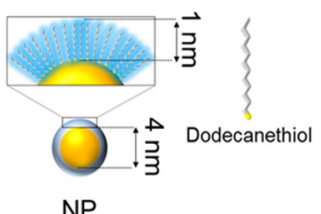

NP b

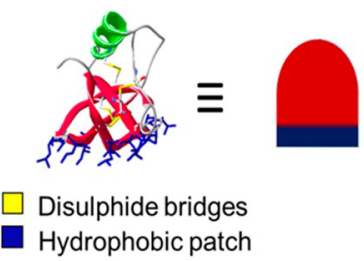

C

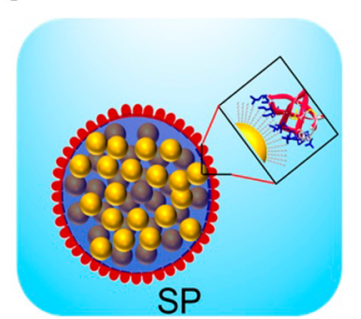

Figure 1. SP structure and starting building blocks. (a) DT-AuNPs used as building blocks for SP self-assembly: yellow represents the AuNP and blue the dodecanethiol stabilizing shell. (b) Cartoon sketching HFBII molecular structure derived from the PDB file 2B97 (left) and its relative schematic representation (right). Color code of the protein secondary structure: red $(\beta$ sheet) and green ribbon $(\alpha$ helix), while the amino acids of the hydrophobic patch are depicted in blue. (c) Self-assembly of DT-AuNPs into a SP is driven by the formation of a HFBII film at the interface where the HFBII hydrophobin patch interacts with the DT ligands on the AuNP surface.

a

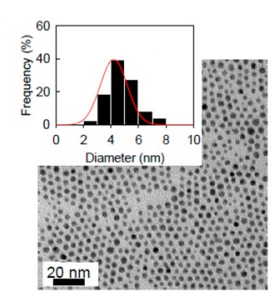

d

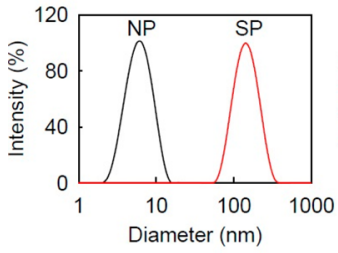

b

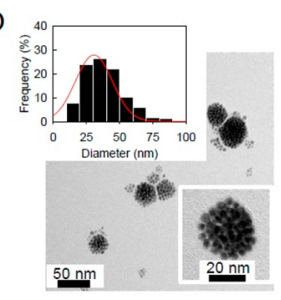

e

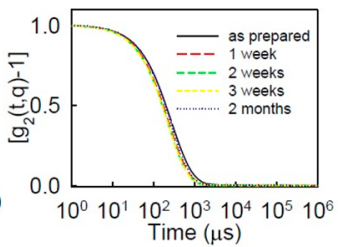

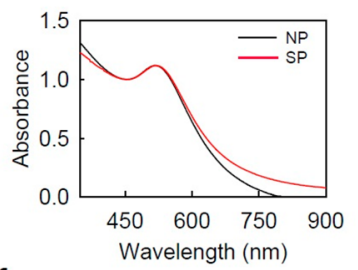

f

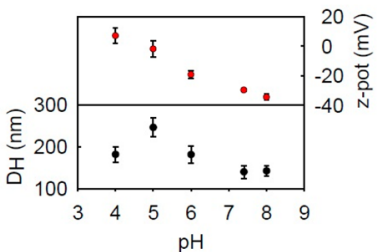

Figure 2. Physical-chemical properties of the self-assembled SPs. (a) Representative TEM image of DT-AuNPs and corresponding size distribution obtained by the analysis of around 100 NPs. (b) Representative TEM image of a dried water dispersion of SPs with the corresponding size distribution obtained by the analysis of around 100 SPs. The inset reports a TEM magnification of one SP. (c) Normalized UV-vis spectrum of chloroform dispersed DT-AuNPs (black line) and water dispersed SPs (red line). (d) Normalized DLS intensity-weighed size distribution of chloroform dispersed DT-AuNPs (NP, black line) and water dispersed SPs (SP, red line). (e) Normalized DLS autocorrelation function curves of water dispersed SPs measured over a time course of two months from their preparation. The plot highlights the kinetic stability of the SP dispersion. (f) Evaluation of the SP physicochemical stability over a pH scan: Dependence of the Z-pot (top of the plot) and the SP size (bottom of the plot) as a function of the $\mathrm{pH}$ of the buffer used to disperse the SPs. Error bars represent the standard error evaluated on six measurements.

polymers, or proteins, which drive the self-assembly in solution as a result of a subtle balance of noncovalent interactions, such as hydrogen bonding, Coulombic and van der Waals (vdW) interactions, and solvophobic effect. ${ }^{9,15,16}$ However, programming hierarchical structure into nanoscale components remains a formidable challenge for nanoscientists.

Several SPs reported in the literature were obtained through controlled aggregation of water-soluble NPs with biomolecules. $^{7,14,17}$ Formation of hybrid SPs composed of hydrophobic NPs in aqueous solutions has also been obtained, exploiting the amphiphilic nature of lipids, ${ }^{18-20}$ surfactants, ${ }^{21,22}$ and polymers. ${ }^{10,23,24}$ In this latter case, the assembly is driven by the hydrophobic effect with formation of defined SPs constituted of a core of assembled hydrophobic NPs coated by an amphiphilic molecule. While lipid vesicles containing NP clusters generally need purification procedures for obtaining homogeneous samples, polymers and surfactants give uniform SPs through simple methodologies (high yield and low cost), ${ }^{10,25,26}$ but often the constituents are poorly metabolized and promote immunological responses. ${ }^{27} \mathrm{~A}$ promising alternative for obtaining biocompatible SPs through simple preparation methods is represented by the use of Janus proteins, that is, biomolecules endowed with a confined highly hydrophobic patch. These biosurfactants may act as template agents that spontaneously assemble hydrophobic NPs in aqueous solutions forming well-defined SPs, which result to be biocompatible and partially biodegradable. ${ }^{14}$

Hydrophobins are small fungal amphiphilic proteins with remarkable surface activity. ${ }^{28}$ Hydrophobin HFBII, obtained from Trichoderma reesei, forms rigid and impermeable films at interfaces preventing the leakage of volatile oils into water, even at high temperature. ${ }^{29}$ It has also been shown that HFBII films prevent immune recognition. ${ }^{30-32}$ Considering that HFBII may form a solid robust film on hydrophobic NPs dispersed in water solution, we studied the self-assembly of dodecanethiol (DT)stabilized AuNPs into aqueous solutions in the presence of HFBII. A class of SPs that showed low cytotoxicity and exceptional stability, even in biological fluids, has been synthesized and characterized. The obtained SPs not only efficiently encapsulated and protected hydrophobic drug molecules by means of the HFBII film but also specifically released their payload intracellularly by glutathione reduction of 
the protein film. This selective mechanism of release has been demonstrated through both in vitro and in vivo studies indicating the suitability of the developed SPs $s$ for future applications in diagnosis and therapy.

\section{RESULTS}

Supraparticle Self-Assembly. We have devised a strategy to prepare water-dispersible and biocompatible SPs composed of a core of hydrophobic DT-protected AuNPs (DT-AuNPs) confined by a HFBII shell (see Figures 1 and S1). In fact, we exploited the surfactant properties of $\mathrm{HFBII}^{33-35}$ to transfer and disperse DT-AuNPs from hydrophobic solvents into aqueous solutions. Briefly, the optimized strategy consisted of a one-pot procedure starting from a two-phase system, HFBII@ water/DT-AuNP@chloroform, which was first completely dried to form a hybrid film and subsequently rehydrated with formation of the desired SPs (details in the methodology section of the SI).

Interfacial surface tension measurements confirmed the ability of HFBII to decrease the energy both at the water/ chloroform and water/DT-AuNP@chloroform interfaces by formation of a protein film (Figure S2). Moreover, a kinetic study showed that, during the initial two-phase step, formation of SPs in the aqueous solution was almost immediate when the two phases came into contact and NP concentration in the aqueous phase increased continuously (Figure S3). These findings suggest that SP formation is driven by an emulsification process, and solvent droplets containing DTAuNPs are stabilized at the interface by the HFBII film. Successive removal of the solvent did not alter the SP structure that kept the initial arrangement upon rehydration (see preparation methodology below).

The structural characterization of the obtained SPs is reported in Figure 2a-g. TEM analysis showed the presence of spherical-like nanocontainers with a mean diameter of around $30 \mathrm{~nm}$ filled with an assembly of DT-AuNPs (Figure 2a,b). These dispersions were studied by a multiangle dynamic light scattering (DLS) analysis resulting in an averaged hydrodynamic diameter $\left(\left\langle D_{\mathrm{H}}\right\rangle\right)$ of $112 \mathrm{~nm}$ (Figure S4) and UV-vis spectroscopy showing a characteristic localized surface plasmon resonance (LSPR) peak centered at $520 \mathrm{~nm}$ (Figure $2 \mathrm{c}, \mathrm{d})$. In Figure $2 \mathrm{e}$ the invariance of DLS autocorrelation functions over two months indicates the high colloidal stability of these dispersions. Colloidal stability (Figure 2f) was also evaluated by DLS and zeta-potential (Z-pot) analyses as a function of the $\mathrm{pH}$ : The formed SPs were dispersed in different buffer solutions at the same ionic strength ranging from $\mathrm{pH} 4$ to 8 .

The dispersions showed a decreased colloidal stability at $\mathrm{pH}$ $=5$ caused by a drop of the Z-pot close to 0 with a subsequent slight agglomeration. This behavior is due to HFBII that at its $\mathrm{pI}$ (isoelectric point), at $\mathrm{pH}=5.2$, is in its zwitterionic form with a net charge close to $0 .{ }^{36}$ Overall, these data highlight the high colloidal stability of the prepared SPs as well as their good compatibility with physiologic $\mathrm{pH}$, which is important for biomedical purposes.

While TEM analysis did not highlight the presence of the protein coating due to the low contrast of the organic material, electron energy loss spectroscopy (EELS) measurements enabled us to evaluate the chemical composition of the hybrid system (Figure 3a). EELS pseudocolor images (Figures $3 \mathrm{~b}-\mathrm{d}$ ) of the corresponding TEM pictures revealed the distribution of nitrogen (red), carbon (green), and gold elements on the SP a

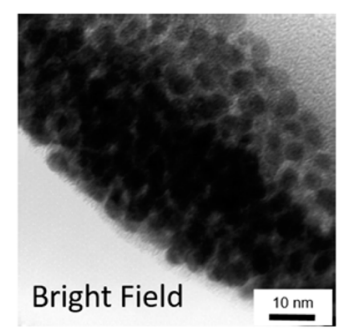

b

C

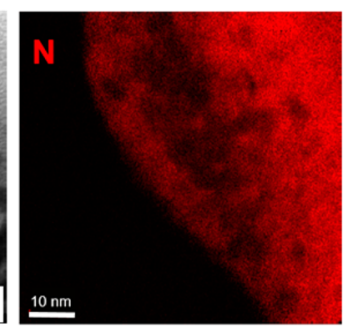

d
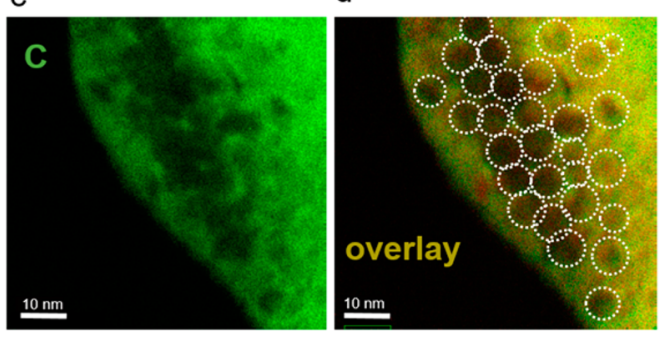

e

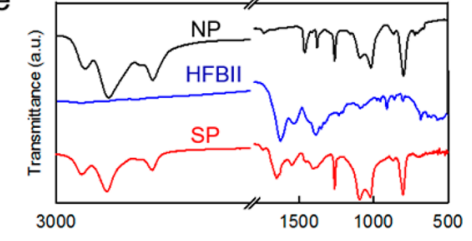

f

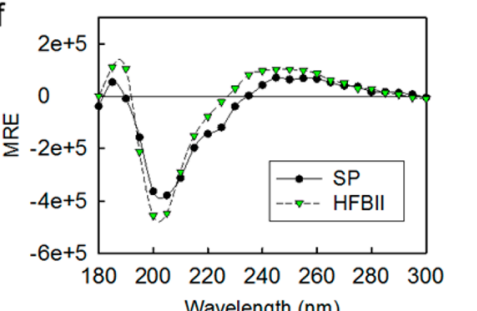

Figure 3. Structural characterization of the SP bionano interface. (a) High-resolution TEM image obtained after drying a SP water dispersion on a TEM holey grid. $(b-d)$ EELS elemental mapping of the SP surface: (b) nitrogen ( $N$, red), (c) carbon ( $C$, green), and (d) overlay of the nitrogen and carbon signals (yellow). The darker spots in (b,c) highlighted as dashed circles in (d) are AuNPs. (e) FTIR spectra of DT-AuNPs (black line), dried HFBII from a 0.1 $\mathrm{mg} / \mathrm{mL}$ water solution (blue line), and SP (red line). The spectrum of the SP sample is a combination of the signals of DT-AuNPs and HFBII spectra. (f) CD spectra of a water solution of HFBII (green dots, $1 \mu \mathrm{M}$ ) and a water dispersion of SPs containing a comparable amount of HFBII (black dots).

surface (circled dark SPs in the $\mathrm{N}-\mathrm{C}$ overlaid picture). The good overlay of the EELS signals attributed to the $\mathrm{N}$ and $\mathrm{C}$ elements emphasizes the protein shell around the selfassembled DT-AuNPs. This was also confirmed by FTIR analysis that showed characteristic peaks of the protein in the $\mathrm{SP}$ spectrum (Figure 3e). More information on the secondary structure of HFBII in the layer surrounding the SPs was obtained by circular dichroism (CD). CD spectra of the SP dispersions highlighted that the formation of the HFBII film was accompanied by a change in the secondary structure of the protein compared to pure HFBII, shown by a decrease in intensity of the peak at $200 \mathrm{~nm}$ and appearance of an absorption band at $225 \mathrm{~nm}$ (black curve in Figure 3f), as expected when the protein forms a film at a hydrophobic/ hydrophilic interface. ${ }^{37}$ Furthermore, the obtained SP dispersions could be lyophilized and redispersed in water 
a

C
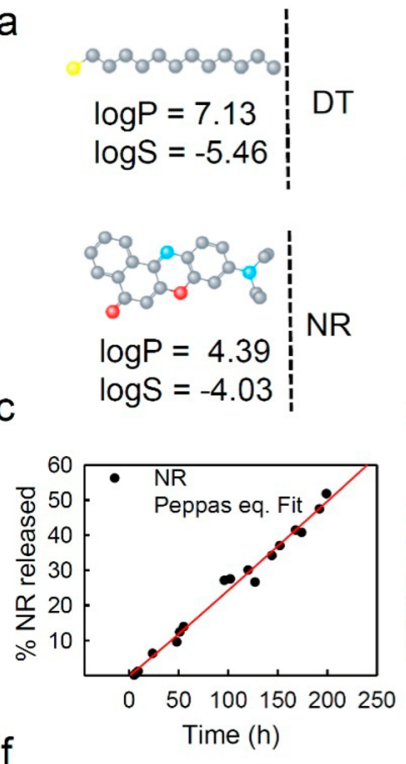

f

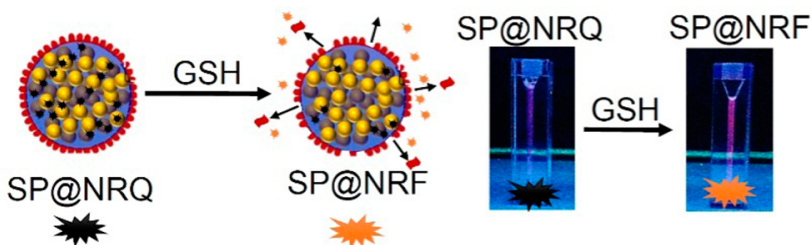

b

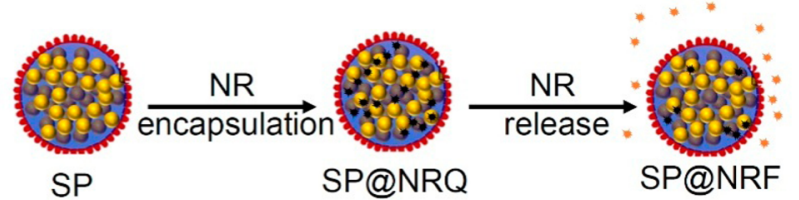

$Q=N R$ quenched

$F=N R$ fluorescing

d

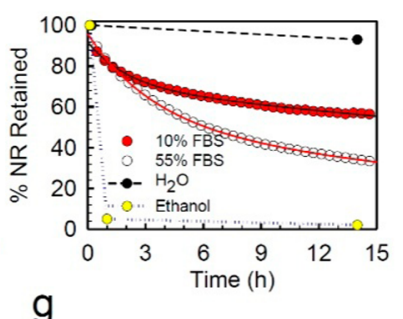

e
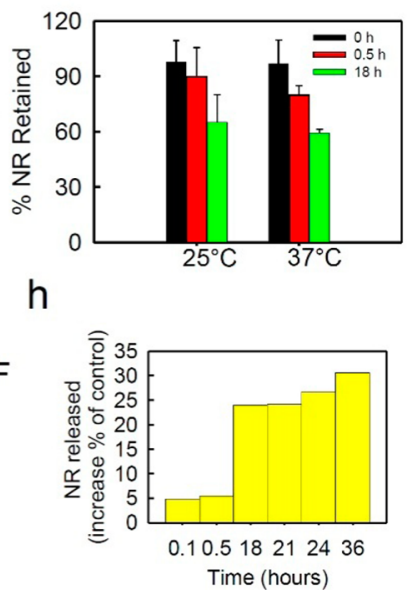

Figure 4. SP ability to encapsulate and release hydrophobic cargo molecules. (a) Chemical structures of DT chains and encapsulated fluorescent molecule NR. Carbon atoms are represented in gray, nitrogen in blue, oxygen in red, and sulfur in yellow. (b) Schematic sketch of the mechanism to detect the release of the fluorescent cargo molecules from the SP in biological fluids: Encapsulation of NR leads to quenching (SP@NRQ) of its fluorescence. In the presence of biological fluids, the potential partition of NR from the SP (SP@NRF) to the surrounding environment leads to a restoration of NR fluorescence that can be monitored. (c) In vitro release experiments in sink conditions. NR-loaded SPs were dialyzed versus $2 \%(\mathrm{w} / \mathrm{V})$ BSA solution over time. The release profile was obtained by measuring NR fluorescence $\left(\lambda_{\mathrm{Ex}}=\right.$ $535 \mathrm{~nm}, \lambda_{\mathrm{Em}}=630 \mathrm{~nm}$ and fit by a Peppas release model (red line). (d) Representative measurements of NR release dynamics in FBS by fluorescence experiments. The normalized (Norm) NR fluorescence represents the amount of NR still associated with SPs after incubation in 10\% FBS (red circles), 55\% FBS (blue circles), water (black circles), or ethanol (yellow circles), respectively. Release kinetics data were fit by a two-compartment model (black and red solid lines). (e) Temperature dependence of the NR release in 10\% FBS. (f) Scheme showing the release of NR in response to SP incubation with GSH and corresponding change of the fluorescence properties of the SP dispersions. (g) Color evaluation of SP@NRQ dispersions upon incubation with GSH (SP@NRF). The cuvette was illuminated under an UV lamp $\left(\lambda_{\mathrm{Ex}}=340\right.$ $\mathrm{nm}$ ). (h) The \% increase of NR fluorescence signal of a SP@NRQ dispersion incubated with GSH with respect to the control SP@NRQ incubated in GSH free medium.

maintaining their initial morphology (Figure S5), thus offering the possibility to be stored as powders rather than in solution. Storage of biomedical formulations as powder is generally preferred, as in this form they are better preserved and for longer time. $^{38}$

Supraparticle as Nanocontainers: Behavior in Biological Fluids. The possibility of using these SPs as potential biomedical nanocontainers depends on their colloidal stability and ability to encapsulate and release hydrophobic molecules in physiological conditions as well as in biological fluids. In order to prove SP colloidal stability in biological fluids, we prepared SP dispersions in cell culture media (DMEM) containing 10\% fetal bovine serum (FBS) and in plasma obtained from healthy mice. These dispersions were stable at DLS analysis for over 96 hours (h) at $37{ }^{\circ} \mathrm{C}$ (Figure S6). TEM analysis of the same samples indicated that the SP structure was preserved also after incubation in the biological milieu (Figure S6).

Having obtained a stable single nano-object that could simultaneously bear a high amount of Au (potential diagnostic purpose) and an extended confined hydrophobic environment, we next aimed at effectively encapsulating hydrophobic cargo molecules. Thus, we initially encapsulated the hydrophobic fluorescent dye Nile Red (NR) in the core of the SPs (SP@NR, scheme in Figure 4a). FTIR and UV-vis analysis of SP@NR showed the successful encapsulation of the dye (Figure S7), while DLS indicated that the size was similar to that of the unloaded SPs. It is known that proximity of the dye with the AuNPs induces a fluorescence quenching on NR emission, ${ }^{39}$ thus this effect was used to monitor the release of the dye as increase in fluorescence (Figures $4 c-h$ and S8). Initially, we studied the release of NR from SP@NR through dialysis versus a $2 \%(\mathrm{w} / \mathrm{v})$ bovine serum albumin (BSA) solution over 8 days. ${ }^{40}$ The presence of BSA should guarantee a better solubility of the NR and mimic the biological environment.

We observed a slow NR release profile as shown in Figure 4c, which could be fit by a Peppas model ${ }^{41}$ and resulted in a nonFickian diffusion mechanism (super case II transport) with a $t_{1 / 2}=194 \mathrm{~h}$ (details reported in Table S1). The most striking result was that after 8 days, about $50 \%$ of dye was still retained in the SPs. To strengthen these results and demonstrate the efficiency of the developed system as carrier of hydrophobic cargoes, a time-scan fluorescence measurement of the SP@NR 


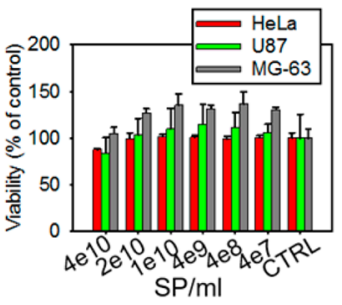

d

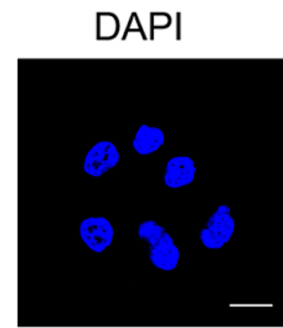

Bodipy

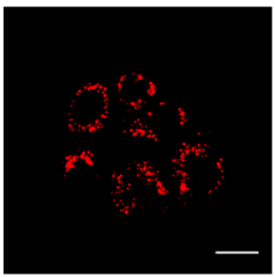

b

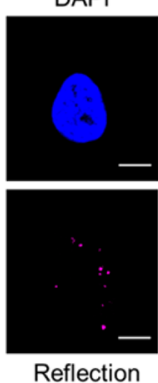

Reflection

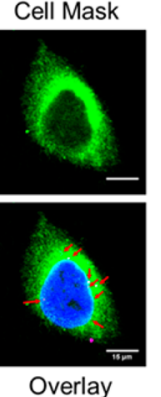

e

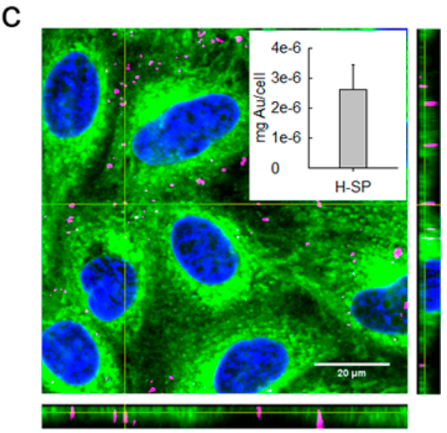

\section{Cell Mask}

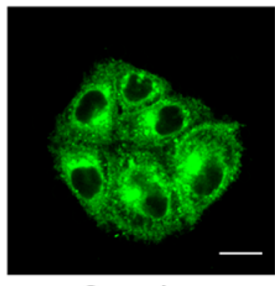

\section{Overlay}
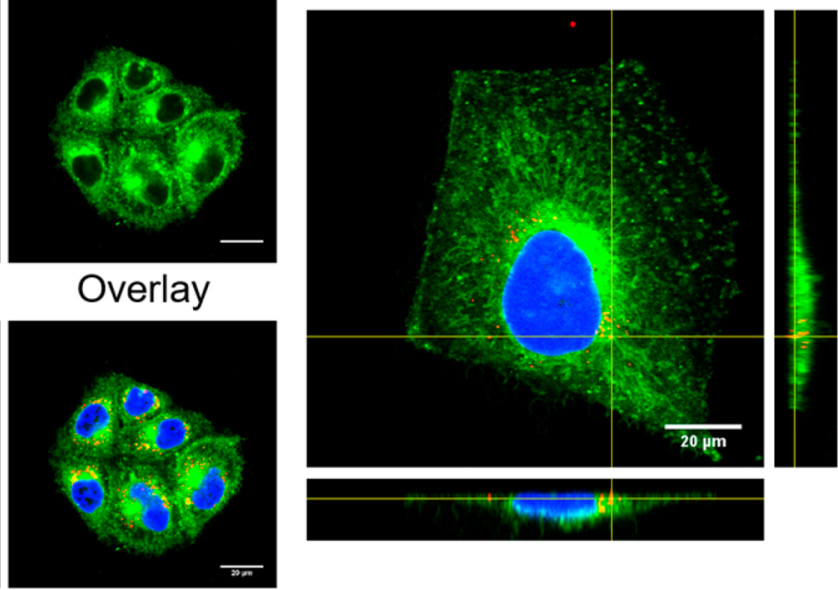

Figure 5. In vitro biological behavior of the SP aqueous dispersions. (a) MTT cell viability test performed incubating different cell lines with increasing concentration of SPs over a time course of $24 \mathrm{~h}$. Untreated cells were used as control. Experiments were carried out in DMEM with $10 \%$ FBS in triplicates. The SP concentration tested in terms of $\mathrm{mg}$ of Au/L were: 10.82, 5.46, 2.73, 1.08, 0.11, and 0.01 mg/L. (b) Evaluation of SP uptake by HeLa: 450,000 HeLa cells have been challenged with $3.8 \times 10^{10} \mathrm{SPs}$ for $24 \mathrm{~h}$ and imaged by CLSM. Cells were stained for cell membrane (Cell Mask) and nucleus (DAPI), while SP were imaged through their scattering properties (reflection). Red arrows indicate the presence of SPs localized in a perinuclear region inside the cell. (c) Orthogonal representation of SP uptake by HeLa cells imaged as describe in (b). The inset reports the SP uptake quantification by ICP-AES in terms of gold content associated with the treated cells. The standard error is calculated on three different replicates. Scale bars are $15 \mu \mathrm{m}$. (d) Evaluation of Bodipy-loaded SP uptake in HeLa cells. Cells were stained and imaged as described in (b), and Bodipy was imaged in the far-red channel. Scale bars are $20 \mu \mathrm{m}$. (e) Single cell confocal microscopy and orthogonal representation of a HeLa cell treated with Bodipy-loaded SP. Scale bar is $20 \mu \mathrm{m}$.

overnight was performed in different environments: water, $10 \%$ FBS, and 55\% FBS at both 25 and $37^{\circ} \mathrm{C}$ (Figures 4d,e). While almost no loss of NR was observed after $18 \mathrm{~h}$ in water, as expected, the presence of proteins in solution promoted NR release. ${ }^{6}$ The curves were analyzed with a two-compartment decay model ${ }^{6}$ resulting in a remaining NR cargo of about $60 \%$ and $35 \%$ of the initial value after $18 \mathrm{~h}$ in $10 \%$ and $55 \%$ FBS, respectively (details in Table S2 and in the SI). The temperature did not show major effects on the release. These results indicated that loaded SPs have an exceptional efficiency in retaining hydrophobic cargoes in biological milieus if compared to other nanocarriers. ${ }^{6,42,43}$ It is likely that the HFBII impermeable and rigid film coating the SPs is responsible for the delayed release of the encapsulated molecules.

Intrigued by the possibility to trigger the sudden release of the drug cargo in vivo by disrupting the HFBII film, we studied the stability of the SPs in reducing environment. In fact, because the SP bionano interface mostly formed by HFBII film (only a tiny protein corona formed in serum, data not shown), the reducing intracellular environment could strongly affect its integrity. In particular, glutathione $(\mathrm{GSH})^{44,45}$ (intracellular concentration 5-10 $\mathrm{mM}$ ) reduces the internal disulfide bridges of HFBII, thus changing its secondary structure (Figure S9) and most probably affecting its film-forming ability.

Empty SPs were incubated in a solution of BSA $(10 \mathrm{mg} / \mathrm{mL})$ with increasing GSH concentrations in a range comparable to its intracellular values and monitored by UV-vis over time. The GSH threshold activation concentration at which SP disassembly starts is between 0.5 and $1.0 \mathrm{mM}$, as demonstrated by the sharp decrease of the plasmon intensity and appearance of a precipitate in the SP aqueous dispersion. The color of these dispersions significantly changed (Figure S10) from purple to light gray with formation of a dark precipitate (uncoated DTAuNPs). UV-vis and DLS analyses of the SP aqueous dispersion incubated with GSH showed the disappearance of the plasmon peak and formation of aggregates, respectively (Figure S9). These results are explained by a progressive dismantling of the HFBII film with loss of stability of the SPs and exposure of their hydrophobic content (DT-AuNPs) into the aqueous solution, which led to aggregation and precipitation of the so-formed AuNP aggregates (Figure S11). This mechanism, taking place also in a biological milieu, may 
potentially occur in vivo, endowing these SPs with an intrinsic and selective intracellular degradation pathway.

Moreover, an additional evidence was obtained incubating SP@NR at $37{ }^{\circ} \mathrm{C}$ with $10 \mathrm{mg} / \mathrm{mL}$ of BSA in the presence of 5 $\mathrm{mM}$ GSH (above the threshold) along a kinetic time course. Fluorescence emission experiments showed that samples treated with GSH (also displaying a different color with respect to the untreated samples) were characterized by an increased fluorescence emission linked to an augmented release of NR. This was also confirmed with stability studies by incubating the SPs with $3 \mathrm{mM} \mathrm{GSH}$ at $37{ }^{\circ} \mathrm{C}$ for $24 \mathrm{~h}$.

Pharmacological and Pharmacokinetic Properties of the SPs in in Vitro and in Vivo Conditions. The biocompatibility of the empty SPs was evaluated by measuring the possible toxicity in five different cell lines (human cervical adenocarcinoma HeLa, human osteosarcoma MG-63, human glioblastoma U-87 MG, triple negative human breast adenocarcinoma MDA-MB-231, and triple negative murine breast adenocarcinoma 4T1) as a function of the SP concentration. No relevant alteration of the viability was observed in all cell lines (>80\%) (Figures 5a and S12). Confocal laser scanning microscopy (CLSM) experiments on HeLa cells treated with the highest concentration used in the cytotoxicity experiments revealed that SPs were internalized in the cell cytoplasm (Figure 5b,c). In particular, the SP appeared localized within vesicular structures accumulating in the perinuclear region consistently with their recruitment into endolysosomal structures (Figure $5 b, c) .{ }^{43}$ SP uptake in HeLa cells was evaluated by ICP-AES as internalized Au content and $24 \mathrm{~h}$ incubation resulted in $10 \%$ of the administered SP dose taken up by the cells (inset in Figure 5c). Cellular uptake of loaded SPs with the hydrophobic fluorophore Bodipy (SP@ Bodipy, Figures S13-S14 for their characterization and release kinetics upon incubation with GSH) was evaluated in HeLa cells as fluorescence emission of the encapsulated dye as well (Figures S12-S13). In particular, HeLa cells incubated with SP@Bodipy for $24 \mathrm{~h}$ (Figure 5d,e) showed both intracellular internalization of the dye in the perinuclear region and a blurry cytosolic localization. While a dotted-like perinuclear pattern is generally consistent, as seen above, with encapsulation inside the endosomes, a cytosolic homogeneous fluorescence usually appears when the dye is already released from the SPs.

To validate this hypothesis, we performed experiments by administering to the cells Bodipy solutions in DMSO. In this case, CLSM analysis reported a different intracellular localization of the free fluorophore that, upon crossing the cell membrane by passive diffusion, seemed to be homogeneously localized in the cytosolic compartment (see Figure S15). To better elucidate the release mechanism of the dye from SP@ Bodipy, we used HEK293-GFP cell lines stably expressing a cytosolic form of the green fluorescent protein (GFP, Figure S16). These cells were incubated with SP@Bodipy for $24 \mathrm{~h}$ and fluorescence microscopy analysis of the sample highlighted a partial intracellular release of the dye as appearance of yellow areas inside the cells due to colocalization of the GFP with free Bodipy signals. However, red-dotted areas due to the presence of SP@Bodipy were also present in the perinuclear region, indicating a delayed and prolonged release of the fluorophore over time.

Having demonstrated the ability of the loaded SPs to intracellularly deliver hydrophobic cargoes, we prepared and characterized SPs containing a prototypic hydrophobic anticancer drug, paclitaxel (PTX) (SP@PTX) (Figure S13).
SP@PTX release kinetics in BSA solutions, also in the presence of GSH, were measured and resembled the behavior displayed by those containing the fluorescent cargoes (Figure S13). The possible cytotoxic effects of the SP@PTX were investigated with respect to the free drug on PTX-sensitive (Hela, MDAMD231) and PTX-resistant (4T1) cancer cells. ${ }^{46,47}$ Encapsulation of the PTX in the SPs resulted in 1 or 2 orders of magnitude reduction of the drug $\mathrm{IC}_{50}$ with respect to the free drug (Figures $6 \mathrm{a}-\mathrm{d}$ and S10-14). Moreover, due to the progressive drug release from the SP core, we observed a clear time-dependent effect of the PTX only when loaded in SPs (Figure S18). a
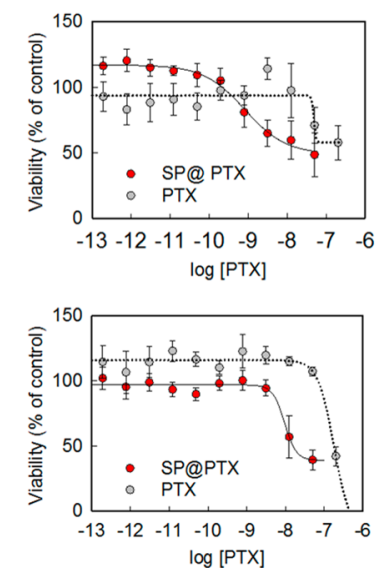

b

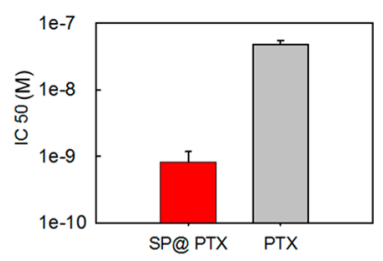

d

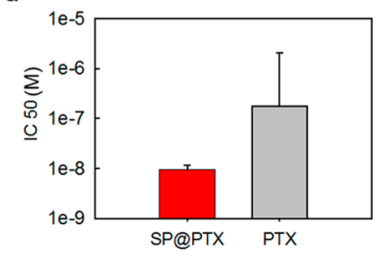

Figure 6. Pharmacological profile of the hydrophobic drug molecule PTX encapsulated in the SPs. (a) Dose-response cytotoxic effect evaluated through an MTT assay on MDA cells incubated with PTX-loaded SPs (SP@PTX) and free PTX (PTX) after a pharmacological treatment of $48 \mathrm{~h}$. Data points are the mean, and standard errors are obtained on six experimental replicates. (b) SP@PTX and PTX $\mathrm{IC}_{50}$ as evaluated from experiments in (a). (c) Dose-response cytotoxic effect evaluated through an MTT assay on 4T1 cells incubated with PTX-loaded SPs (SP@PTX) and free PTX (PTX) performed after a pharmacological treatment of $24 \mathrm{~h}$. Data points are the mean and the standard errors obtained from six experimental replicates. (d) SP@PTX and PTX IC s0 $_{0}$ as evaluated from experiments in (c).

The assessment of the interaction between tumor cells and the developed SPs is essential for their application as diagnostic or therapeutic tools. However, in vitro efficacy is not sufficient to prove the in vivo effectiveness of a nanocarrier, as to exert its pharmacological activity, the nanocarrier has to be stable in the in vivo biological environment before reaching the pathological target. $^{6}$

In the perspective of using these SPs for biomedical applications either as diagnostic or therapeutic tools, longitudinal analyses of both in vivo biodistribution (using SP@ Bodipy) and pharmacokinetics (using SP@PTX) were performed in healthy mice after intravenous (iv) administration. First, the fate of SP@Bodipy, both considering gold and loaded dye, was evaluated through in vivo and ex vivo studies (Figures 7). Figure 7a shows serial scans of the same mouse treated with SP@Bodipy at different time-points. Very interestingly, a progressive increase of the signal was observed. This is apparently in contrast with the largest part of the experiments of optical imaging performed with fluorescent dyes, in which clearance and excretion lead to a time-dependent 


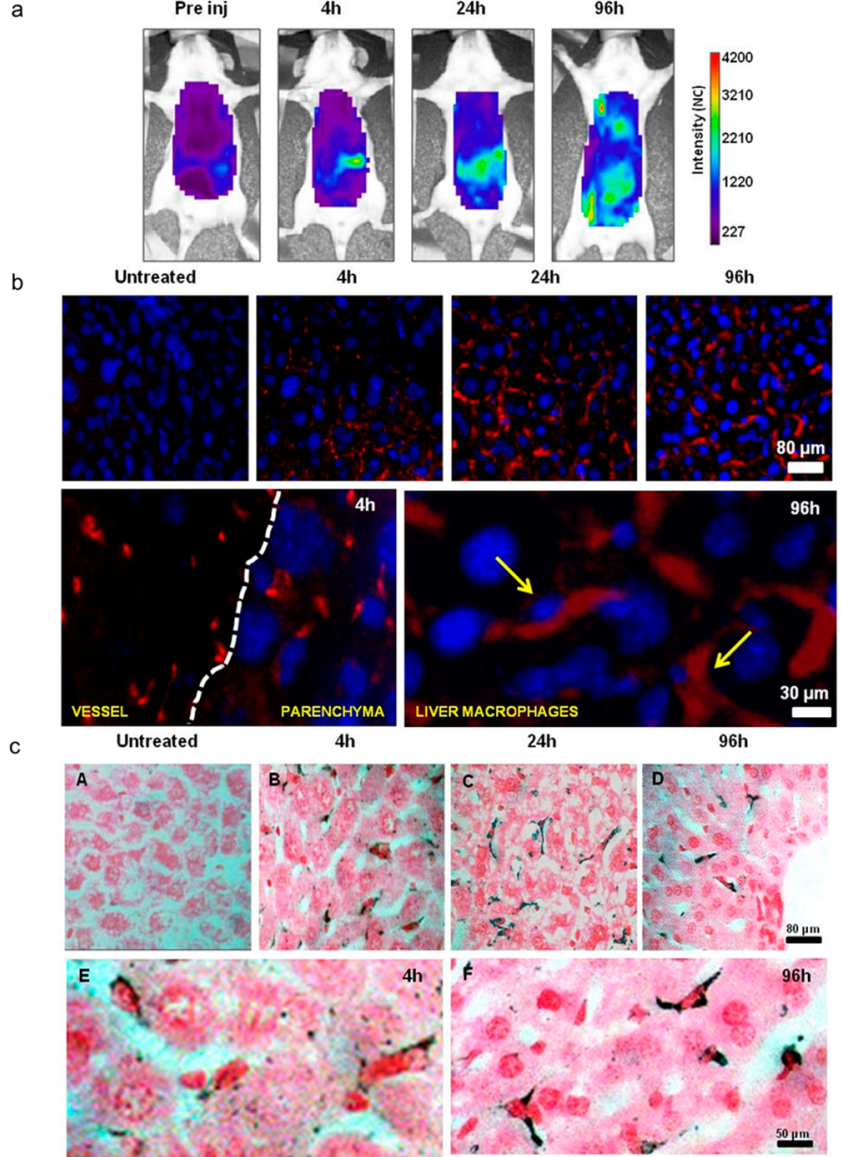

Figure 7. (a) In vivo biodistribution of SP@Bodipy after iv administration in the same mouse. The fluorescence signal intensity, measured as normalized photon counts (NC), is shown as a pseudocolor scale bar. The scale bar is consistent for all images. (b) Liver sections from untreated and SP@Bodipy-treated mice sacrificed 4, 24, $96 \mathrm{~h}$, respectively. In blue, the nuclei were stained with Hoechst 33258, and in red, the signal is associated with Bodipy. (C) Liver sections from untreated and SP@Bodipytreated mice sacrificed 4, 24, $96 \mathrm{~h}$, respectively. Black spots are representative of the gold accumulation revealed by silver lactate, and the parenchyma was revealed by Safranin $O$ staining.

reduction of the signal. Here, considering that gold has a quenching effect on the emission of the dye (as demonstrated in the previous in vitro experiments), the initial increase in fluorescence $(24 \mathrm{~h})$ is interpreted as due to the disassembling of the SP@Bodipy supraparticles once they have reached the organs and penetrated into the cells. Moreover, dissociation between gold (carrier) and Bodipy (cargo) is mainly associated with the abdominal region until $24 \mathrm{~h}$ after the treatment, and at the $96 \mathrm{~h}$, it spreads in different regions, including lymph nodes and thoracic and pelvic areas. The actual migration of the SP@ Bodipy in filter organs is demonstrated by comparing Figure $7 \mathrm{~b}, \mathrm{c}$. In Figure $7 \mathrm{~b}$ representative images of liver sections stained with the nuclear markers Hoechst 33258 (blue) are reported. The upper panel depicts a progressive increase of Bodipy (red staining) in these sections until $24 \mathrm{~h}$ after the treatment, followed by a decrease at the last time point. This trend is in agreement with in vivo optical imaging and furthermore confirms the hypothesis of a disassembling of the adduct gold-dye inside the organ. Higher magnified pictures of the liver parenchyma at 4 and $96 \mathrm{~h}$ after treatment, respectively, are reported in the lower panel of Figure $7 \mathrm{~b}$. The presence of red staining around the nuclei was observed at both time points, suggesting that an active internalization inside the cells is required to detect Bodipy fluorescent signal. The main difference is the anatomical localization of the dye; in the liver of the mouse sacrificed $4 \mathrm{~h}$ after the treatment, it is almost homogeneously diffused, whereas $96 \mathrm{~h}$ after the administration, it is confined in ramified and polarized cells with smaller nuclei that very likely belong to the Kupffer cells. The accumulation of gold in the Kupffer cells, investigated through histological analysis by autometallography (AMG) (Figure 7c, lower panel, right) strongly suggests that intact SP@Bodipy enters in the cells and then disassembles, allowing the release of the dye and consequently the detection of fluorescence. Figure $7 \mathrm{c}$, upper panels, shows the kinetics of gold accumulation in liver (black spots) in full agreement with the fluorescent trend: The highest level of the signal is after $24 \mathrm{~h}$ with a progressive reduction over time. Moreover, gold accumulation was not homogeneously spread in the whole parenchyma, but it seemed more localized in thin and ramified cells (most probably macrophages and Kupffer cells). Overall, in vitro and in vivo results obtained using SP@Bodipy suggest that (1) the cargo is efficiently retained into the carrier until its cellular internalization and (2) the presence of a reducing environment (GSH) intracellularly induces disassembly of the complex. Further experiments were conducted in order to evaluate the pharmacokinetics of NP@ PTX in comparison with the classical cremophor-based formulation of PTX in terms of release and biodistribution.

Figure 8a shows the pharmacokinetic profile of PTX in plasma of mice receiving $20 \mathrm{mg} / \mathrm{kg}$ either freely injected in cremophor or loaded into the SPs. The levels of the drug in the bloodstream of the animals treated with SP@PTX were markedly lower than the other group and rapidly decreased disappearing $4 \mathrm{~h}$ after administration. This difference could be due to a rapid distribution of the SP@PTX into the main filter organs. In fact, the levels of PTX recovered in the liver were higher after the administration of SP@PTX than cremophorPTX (Figure 8d). The rapid and strong tropism to the filter organs is furthermore demonstrated by the increase of splenic and pulmonary levels of PTX in the group treated with SPs (Figure S19). This result confirms that the low amount of PTX and gold in the plasma is mainly due to a rapid sequestration of the SPs. To assess that this sequestration was actually related to an active penetration of the SPs in the liver parenchyma, ICPMS measurements were carried out in the same mice, and the trend of PTX and gold SPs was comparable at different timepoints (Figures 8e-f). Similarly to what we found for the liver, the curves of the kinetics of gold and drug in plasma showed a similar profile, strongly suggesting a stable association between PTX and the gold carrier after administration in the systemic circulation.

Even if a single administration of $20 \mathrm{mg} / \mathrm{kg}$ of PTX alone is normally well tolerated, we could not exclude an additive effect played by gold accumulation. To evaluate histopathological alterations, representative $\mathrm{H} \& \mathrm{E}$-stained sections from spleen, liver, and lungs of animals sacrificed $96 \mathrm{~h}$ after treatment with PTX and SP@PTX were compared to those of untreated mice (Figure S20). Our data did neither reveal any macroscopic modification of parenchyma nor features of inflammation and necrosis. As in the case of SP@Bodipy, in vivo studies suggest that SP@PTX supraparticles are stable in vivo, accumulate in filter organs, and are able to release their cargo once penetrated into the cells. 

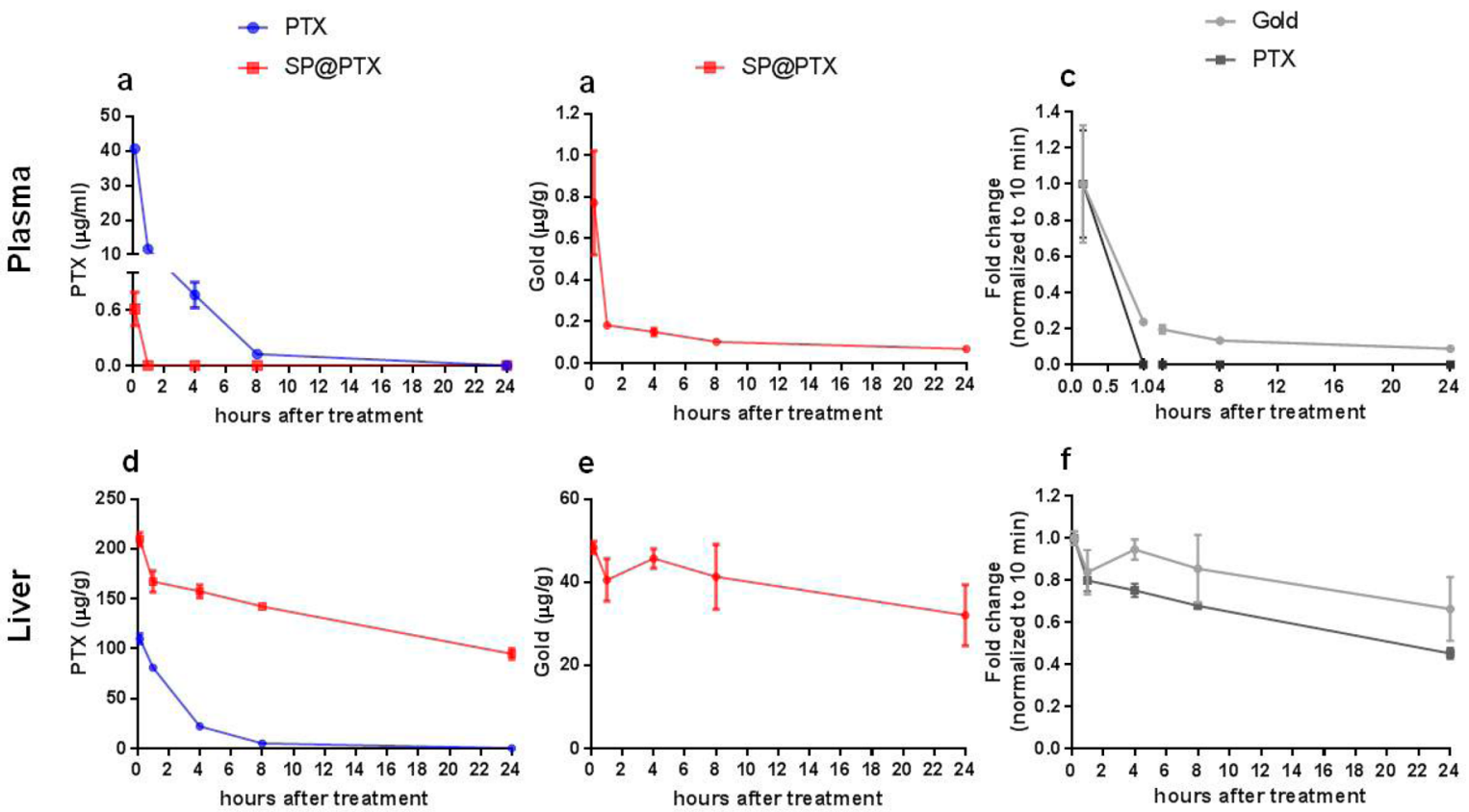

Figure 8. Levels of PTX in (a) plasma and (d) liver measured by HPLC after iv treatment with free PTX or SP@PTX in healthy mice. Levels of gold in (b) plasma and (e) liver measured by ICP-MS after SP@PTX injection. Each group has three mice as replicates. Data are expressed as mean \pm SE. Two way-ANOVA followed by Bonferroni posthoc test was carried out. Multiple comparisons (matching both treatments and time points) show a significant difference in kinetics and biodistribution of the two formulations. Trend of levels of both gold and PTX in (c) plasma and (f) liver derived from mice treated with SP@PTX. The values were normalized to 1 at the first time point (10 min).

\section{DISCUSSION}

We have developed a facile and efficient methodology, which exploits HFBII surfactant and film-forming properties, to assemble hydrophobic AuNPs into water-stable and biocompatible SPs stable in the biological environment. These waterdispersible SPs possess an extended inner hydrophobic compartment that can be exploited for drug loading. This highly hydrophobic core matches the physicochemical properties (such as hydrophobicity and solvent miscibility) of most conventional chemotherapeutics and is able to avoid the release of hydrophobic drugs in the systemic circulation before reaching tissue parenchyma. ${ }^{6,48}$ In fact, the importance of the cargo-carrier compatibility for reducing undesired release of poorly soluble drugs has been recently demonstrated for polymeric PLGA (poly(lactic-co-glycolic acid) NPs in vivo. ${ }^{6,42}$ Similarly, our release experiments on the developed SPs demonstrated a high ability to retain the cargoes inside the carrier in biological fluids, which represents one of the main hurdles on the targeted delivery of nanodrugs. On the other hand, a sudden release of the drug upon SP uptake by the cells is triggered by the reducing action of the intracellular GSH, which promotes breakage of the HFBII coating. As a consequence, an encapsulated anticancer drug, SP@PTX, showed 2 orders of magnitude decrease of its $\mathrm{IC}_{50}$ in in vitro experiments on target cells. Through an elegant and original approach combining in vivo tracking of the SPs with either a fluorescent dye or a drug, we were able to demonstrate the working hypotheses postulated above. In particular, this study highlights the reliability of the developed system for its intracellular delivery with minimal dispersion of the payload. Although other steps, such as the functionalization of the surface to pass through biological barriers and/or to reach specific targets will have to be considered, we believe that the demonstration of this general behavior may represent a robust starting point to think about a next generation of nanocarriers. The obtained SP represents a theranostic nanosystem able to simultaneously carry high content of AuNPs and drugs, ${ }^{30}$ but also, by means of the impermeable HFBII coating, prevents leakage of the drug. ${ }^{31,32}$ AuNPs were chosen for their easy preparation ${ }^{49}$ and bioinertness, ${ }^{49,50}$ along with the possibility to use the high content of $\mathrm{Au}$ in the SP core as diagnostic and therapeutic tool (in fact, gold provides nearly 3 -fold greater Xray attenuation per unit weight than iodine) in computed tomography and radiation therapy ${ }^{10,51,52}$ applications. The applicability of the developed methodology is general and may be applied to the obtainment of a broad variety of new SPs, for example, fluorinated ones. Furthermore, the possibility to modify chemically or genetically, that is, fusion proteins, ${ }^{53}$ the HFBII shell allows for further functionalization of the SP surface for targeting specific tissues. Overall, these results are important for guiding advances in the design of multistage and multimodal nanosystems for diagnosis and therapy.

\section{METHODS}

Procedure to Prepare the HFBII-Stabilized SPs. $900 \mu \mathrm{L}$ of DTAuNP (stock concentration about $1-3 \times 10^{15} \mathrm{NPs} / \mathrm{mL}$ ) were precipitated using ethanol $(\cong 10 \mathrm{~mL})$ and centrifuged at $8000 \mathrm{rpm}$ for $30 \mathrm{~min}$. The obtained pellet was dried using compressed air, resuspended in $900 \mu \mathrm{L}$ of chloroform and moved in a round bottomed flask. HFBII was obtained as described in reference. ${ }^{54} \mathrm{~A}$ fresh solution of HFBII $0.1 \mathrm{mg} / \mathrm{mL}$ was prepared by dissolving an accurately weighed amount of the protein in Milli- $Q$ water $(m Q w)$ under sonication for $15 \mathrm{~min}$ using a bath. NP dispersion was then placed in a water bath at $50{ }^{\circ} \mathrm{C}$ and stirred magnetically (>300 rpm). The freshly prepared HFBII solution was added dropwise to the NP dispersion and subsequently the NP/HFB biphasic solution was left under magnetic stirring at $50{ }^{\circ} \mathrm{C}$ for $1 \mathrm{~h}$. During stirring $\mathrm{CHCl}_{3}$ and aqueous phases were vigorously mixed and the aqueous phase gradually turned pink, as a consequence of NP transfer into the aqueous phase via SPs formation. The sample was subsequently dried 
using rotavapor. Evaporation was gradually performed at room temperature leading to the formation of a dried film. The dried film was dissolved in $\mathrm{mQw}(10 \mathrm{~mL})$ and subject of ultrasound bath for 15 min. The obtained SP dispersion was left overnight at RT and dialyzed against $\mathrm{mQw}$ for $24 \mathrm{~h}$.

Three 1 min-tip-sonication cycles were performed on the dialyzed sample using a SONIC Vibracell (Newtown, CT) operating at $20 \mathrm{~V}$ and set at $80 \%$ power. For dye and drug encapsulation, the molecule to be encapsulated was dissolved in $200 \mu \mathrm{L}$ of the organic phase $\left(\mathrm{CHCl}_{3}\right)$ and incubated overnight with $800 \mu \mathrm{L}$ of the NP dispersion. After that the encapsulation of the drug and the NPs to form SPs was accomplished following the same procedure explained above to prepare empty SPs. The purification of the drug loaded SPs was performed by centrifugation: first centrifugation run (speed $1000 \mathrm{~g}$ for $20 \mathrm{~min})$; second centrifugation run (5000 $\mathrm{g}$ for $40 \mathrm{~min})$; third centrifugation run ( $5000 \mathrm{~g}$ for $40 \mathrm{~min}$ ). At each purification step the obtained supernatant was collected and analyzed by FTIR and UV-vis to disclose the presence of free HFBII or drug molecule, while the pellet was suspended in Milli-Q water and processed again. In a typical purification process three runs are sufficient to remove the excess free drug in solution. SP number was evaluated as it follows: the volume of a $\mathrm{SP}$ with a radius $15 \mathrm{~nm}$ is $\mathrm{V}_{\mathrm{SP}}=\frac{4}{3} \pi r^{3}$ while the weight of a SP is obtained approximately multiplying its volume to the density of gold $\left(19 \mathrm{~g} / \mathrm{cm}^{3}\right)$. The SP concentration was then obtained by dividing the gold content of the SP preparation as measured by ICP-AES to the approximate weight of a SP.

In vitro drug release characterization. To measure the release pattern of Nile Red from the SP@NR, $2.5 \mathrm{~mL}$ of SP@NR were placed into a dialysis bag (MWCO: $3000 \mathrm{Da}$ ), which was subsequently immersed in $25 \mathrm{~mL}$ of aqueous media containing $2 \%(\mathrm{w} / \mathrm{v}$ ) BSA at 25 ${ }^{\circ} \mathrm{C}$ under gently stirring. At predetermined time intervals, $1 \mathrm{~mL}$ of the equilibrated solution was recovered and the NR content was determined by fluorescence measurement using an excitation of 535 $\mathrm{nm}$ and measuring the emission at $630 \mathrm{~nm}$. The excitation and emission width were fixed at $5 \mathrm{~nm}$ while the sensitivity of the Fluorimeter (Jasco) was set on high. In order to maintain the sink conditions, each $\mathrm{mL}$ of solution recovered was replenished with a fresh one. To evaluate the amount of NR release a calibration curve was used. The drug release data obtained were fitted to a Peppas model using SigmaPlot.

In vivo experiments. Mice were maintained under specific pathogen-free condition in the Institute's Animal Care Facility and regularly checked by a certified veterinarian. Food and water were available for ad libitum consumption. Artificial light was provided in a $12 \mathrm{~h} / 12 \mathrm{~h}$ cycle. Procedures involving animals and their care were conducted in conformity with institutional guidelines in compliance with national (Legislative Decree n. 26, March 4, 2014; Authorization n.19/2008-A issued March 6, 2008, by the Italian Ministry of Health) and international laws and policies (EEC Council Directive 2010/63, August 6, 2013; Standards for the Care and Use of Laboratory Animals, U.S. National Research Council, Statement of Compliance A5023-01, October 28, 2008).

Four-week-old female BALB/c mice (CDI envigo) were enrolled for biodistribution and pharmacokinetics study. Regarding biodistribution, four animals were intravenously treated with $200 \mu \mathrm{L}$ of SP@ Bodipy $\left(1 \times 10^{12} \mathrm{SPs} / \mathrm{mL}\right)$. Optical imaging was conducted on animals before and at 4, 24, and $96 \mathrm{~h}$ after SPs injection using the Explore Optix System (ART, Advanced Research Technologies, Montreal, Canada), as already described by our group. ${ }^{55}$ Selected region of interest (ROI) scan (ventral area) was performed with a step size of $2 \mathrm{~mm}$. After in vivo imaging, mice were sacrificed by decapitation, and the liver was collected and immediately frozen for histological analysis to visualize both Bodipy and gold accumulation.

As regards pharmacokinetics, animals were treated with $20 \mathrm{mg} / \mathrm{kg}$ of cremophor PTX $(n=15)$ or SP@PTX $(n=15)$. Blood was collected, in heparinized tubes, $10 \mathrm{~min}, 1,4,8$, and $24 \mathrm{~h}$ after treatment and centrifuged to obtain plasma. After mice sacrifice, liver, spleen, and lungs were collected and stored at $-20{ }^{\circ} \mathrm{C}$ until ICP-MS analysis (to detect the presence of gold) and HPLC-UV (to measure the levels of PTX). Elemental analysis of organs was carried out by ICP-MS as described before (methods in the SI) to evaluate the Au content. To measure its concentration, each organ was weighed before the analysis. The total concentration of PTX in the different biological matrices was determined by HPLC as previously described. ${ }^{56}$ For the determination of PTX in tissues and organs, they were previously homogenized in $0.2 \mathrm{M} \mathrm{CH}_{3} \mathrm{COONH}_{4} \mathrm{pH}$ 4.5.

Each study sample $(0.3 \mathrm{~mL}$ for plasma and $0.5 \mathrm{~mL}$ for homogenate tissues) was assayed together with a five points of standard calibration curve prepared in the corresponding control biological matrix obtained from untreated mice at concentrations ranging from 0.05 to $5 \mu \mathrm{g} /$ sample. The limits of quantification (LOQ) were $0.16 \mu \mathrm{g} / \mathrm{mL}$ and 0.6 $\mu \mathrm{g} / \mathrm{g}$ for plasma and organs, respectively

Histological Studies. Histological analyses were performed on sections derived from mice treated with SP@Bodipy. Fluorescent analysis was performed incubating liver cryostatic sections $(30 \mu \mathrm{m}$ of thickness) with the vital nuclear dye Hoechst $33258(2 \mu \mathrm{g} / \mathrm{mL}$ in PBS) and then acquired by Olympus Fluoview microscope BX61 (Tokyo, Japan) with confocal system FV500 equipped by specific lasers $\lambda_{\text {exc }}=405 \mathrm{~nm}$ for Hoechst 33258 and $\lambda_{\text {exc }}=635 \mathrm{~nm}$ to visualize the specific signal associated with Bodipy.

Liver micrometric sections $(4 \mu \mathrm{m})$ were cut to analyze the metal tissue distribution, and autometallography staining was carried out by as recently described. At the end of the staining, sections were visualized through a light microscope for the identification and localization of gold aggregates, visible as black granular pigments. ${ }^{57}$

\section{ASSOCIATED CONTENT}

\section{Supporting Information}

The Supporting Information is available free of charge on the ACS Publications website at DOI: 10.1021/acsnano.7b04979.

An extended discussion about SPs formation as well as supplementary figures and methods are presented (PDF)

\section{AUTHOR INFORMATION}

\section{Corresponding Authors}

*E-mail: pierangelo.metrangolo@polimi.it.

*E-mail: francesca.baldelli@polimi.it.

ORCID $\odot$

Claudia Pigliacelli: 0000-0002-0143-9045

Gabriele Candiani: 0000-0003-0575-068X

Paolo Bigini: 0000-0002-0239-9532

Pierangelo Metrangolo: 0000-0002-7945-099X

Francesca Baldelli Bombelli: 0000-0001-8138-9246

\section{Present Addresses}

${ }^{\perp}$ Hyber Center of Excellence, Department of Applied Physics, Aalto University, Puumiehenkuja 2, FI-00076 Espoo, Finland.

${ }^{\nabla}$ Nanobiointeractions and Nanodiagnostics, Istituto Italiano di Tecnologia, Via Morego 30, Genova, Italy.

\section{Author Contributions}

"These authors contributed equally to this work. D.M., C.P., P.M., and F.B.B. designed the study. P.M. and F.B.B. supervised the study. D.M., C.P., and P.S.M. performed the SP assembly and encapsulation studies. D.M. performed the release studies. P.S.M., D.M., L.T. and M.B.V. performed and designed cell experiments. P.B., R.P., and M.Z. designed the in vivo experiments. G.M., L.T., L.M., and R.F. performed the in vivo experiments. I.T. and C.P. performed NP synthesis. G.C. analyzed the data and supplied reagents and equipment.

\section{Funding}

The authors gratefully acknowledge the financial support of Regione Lombardia (Fondo per lo Sviluppo e la Coesione FAS 2007-2013) through Fondazione Centro Europeo di 
Nanomedicina (CEN). P.M. gratefully acknowledges the financial support of the European Research Council (ERC) through the grant FOLDHALO (www.foldhalo.eu), no. 307108 .

\section{Notes}

The authors declare no competing financial interest.

\section{ACKNOWLEDGMENTS}

We would like to thank Andrea Serafini for the TEM measurements and the laboratory of Chemistry for Technology, University of Brescia for giving access to their tensiometer. We are grateful to Maurizio Ursini, Maria Tringali, and Claire Michelet for technical assistance.

\section{REFERENCES}

(1) Torchilin, V. P. Multifunctional, Stimuli-Sensitive Nanoparticulate Systems for Drug Delivery. Nat. Rev. Drug Discovery 2014, 13, 813-827.

(2) Liu, X.; Situ, A.; Kang, Y.; Villabroza, K. R.; Liao, Y.; Chang, C. H.; Donahue, T.; Nel, A. E.; Meng, H. Irinotecan Delivery by LipidCoated Mesoporous Silica Nanoparticles Shows Improved Efficacy and Safety over Liposomes for Pancreatic Cancer. ACS Nano 2016, 10, 2702-2715.

(3) Riehemann, K.; Schneider, S. W.; Luger, T. a; Godin, B.; Ferrari, M.; Fuchs, H. Nanomedicine-Challenge and Perspectives. Angew. Chem., Int. Ed. 2009, 48, 872-897.

(4) Lu, Y.; Aimetti, A. A.; Langer, R.; Gu, Z. Bioresponsive Materials. Nat. Rev. Mater. 2016, 2, 16075.

(5) Wilhelm, S.; Tavares, A. J.; Dai, Q.; Ohta, S.; Audet, J.; Dvorak, H. F.; Chan, W. C. W. Analysis of Nanoparticle Delivery to Tumours. Nat. Rev. Mater. 2016, 1, 16014.

(6) Zhao, Y.; Fay, F.; Hak, S.; Manuel Perez-Aguilar, J.; SanchezGaytan, B. L.; Goode, B.; Duivenvoorden, R.; de Lange Davies, C.; Bjørkøy, A.; Weinstein, H.; Fayad, Z. A.; Pérez-Medina, C.; Mulder, W. J. M. Augmenting Drug-carrier Compatibility Improves Tumour Nanotherapy Efficacy. Nat. Commun. 2016, 7, 11221.

(7) Park, J. Il; Nguyen, T. D.; de Queirós Silveira, G.; Bahng, J. H.; Srivastava, S.; Zhao, G.; Sun, K.; Zhang, P.; Glotzer, S. C.; Kotov, N. a. Terminal Supraparticle Assemblies from Similarly Charged Protein Molecules and Nanoparticles. Nat. Commun. 2014, 5, 3593.

(8) Zhuang, J.; Wu, H.; Yang, Y.; Cao, Y. C. Controlling Colloidal Superparticle Growth through Solvophobic Interactions. Angew. Chem., Int. Ed. 2008, 47, 2208-2212.

(9) Silvera Batista, C. A.; Larson, R. G.; Kotov, N. A. Nonadditivity of Nanoparticle Interactions. Science (Washington, DC, U. S.) 2015, 350, 1242477-1242477.

(10) Al Zaki, A.; Joh, D.; Cheng, Z.; et al. Gold-Loaded Polymeric Micelles for Computed Tomography-Guided Radiation Therapy Treatment. ACS Nano 2014, 8, 104-112.

(11) Yang, Y.-S.; Carney, R. P.; Stellacci, F.; Irvine, D. J. Enhancing Radiotherapy by Lipid Nanocapsule-Mediated Delivery of Amphiphilic Gold Nanoparticles to Intracellular Membranes. ACS Nano 2014, 8, 8992-9002.

(12) Bishop, K. J. M. Hierarchical Self-Assembly for Nanomedicine. Angew. Chem., Int. Ed. 2016, 55, 1598-1600.

(13) Wang, Y.; Wise, A. K.; Tan, J.; Maina, J. W.; Shepherd, R. K.; Caruso, F. Mesoporous Silica Supraparticles for Sustained Inner-Ear Drug Delivery. Small 2014, 10, 4243.

(14) Vargo, K. B.; Zaki, A.; Al; Warden-Rothman, R.; Tsourkas, A.; Hammer, D. A. Superparamagnetic Iron Oxide Nanoparticle Micelles Stabilized by Recombinant Oleosin for Targeted Magnetic Resonance Imaging. Small 2015, 11, 1409-1413.

(15) Maiolo, D.; Paolini, L.; Di Noto, G.; Zendrini, A.; Berti, D.; Bergese, P.; Ricotta, D. Colorimetric Nanoplasmonic Assay to Determine Purity and Titrate Extracellular Vesicles. Anal. Chem. 2015, 87, 4168-4176.
(16) Xia, Y.; Nguyen, T. D.; Yang, M.; Lee, B.; Santos, A.; Podsiadlo, P.; Tang, Z.; Glotzer, S. C.; Kotov, N. A. Self-Assembly of SelfLimiting Monodisperse Supraparticles from Polydisperse Nanoparticles. Nat. Nanotechnol. 2011, 6, 580-587.

(17) Khandelia, R.; Bhandari, S.; Pan, U. N.; Ghosh, S. S.; Chattopadhyay, A. Gold Nanocluster Embedded Albumin Nanoparticles for Two-Photon Imaging of Cancer Cells Accompanying Drug Delivery. Small 2015, 11, 4075-4081.

(18) Wang, T.; Wang, X.; LaMontagne, D.; Wang, Z.; Wang, Z.; Cao, Y. C. Shape-Controlled Synthesis of Colloidal Superparticles from Nanocubes. J. Am. Chem. Soc. 2012, 134, 18225-18228.

(19) Yang, N.; Yang, Z.; Held, M.; Bonville, P.; Albouy, P.-A.; Lévy, R; Pileni, M.-P. Dispersion of Hydrophobic Co Supracrystal in Aqueous Solution. ACS Nano 2016, 10, 2277-2286.

(20) Delcea, M.; Sternberg, N.; Yashchenok, A. M.; Georgieva, R.; Bäumler, H.; Möhwald, H.; Skirtach, A. G. Nanoplasmonics for DualMolecule Release through Nanopores in the Membrane of Red Blood Cells. ACS Nano 2012, 6, 4169-4180.

(21) Bai, F.; Wang, D.; Huo, Z.; Chen, W.; Liu, L.; Liang, X.; Chen, C.; Wang, X.; Peng, Q.; Li, Y. A Versatile Bottom-up Assembly Approach to Colloidal Spheres from Nanocrystals. Angew. Chem., Int. Ed. 2007, 46, 6650-6653.

(22) Lacava, J.; Born, P.; Kraus, T. Nanoparticle Clusters with Lennard-Jones Geometries. Nano Lett. 2012, 12, 3279-3282.

(23) Chen, O.; Riedemann, L.; Etoc, F.; Herrmann, H.; Coppey, M.; Barch, M.; Farrar, C. T.; Zhao, J.; Bruns, O. T.; Wei, H.; Wei, H.; Guo, P.; Cui, J.; Jensen, R.; Chen, Y.; Harris, D. K.; Cordero, J. M.; Wang, Z.; Jasanoff, A.; Fukumura, D.; et al. Magneto-Fluorescent Core-Shell Supernanoparticles. Nat. Commun. 2014, 5, 5093.

(24) Sánchez-Iglesias, A.; Grzelczak, M.; Altantzis, T.; Goris, B.; Pérez-Juste, J.; Bals, S.; Van Tendeloo, G.; Donaldson, S. H.; Chmelka, B. F.; Israelachvili, J. N.; Liz-Marzán, L. M. Hydrophobic Interactions Modulate Self-Assembly of Nanoparticles. ACS Nano 2012, 6, 1105911065 .

(25) He, J.; Huang, X.; Li, Y.-C.; Liu, Y.; Babu, T.; Aronova, M. a; Wang, S.; Lu, Z.; Chen, X.; Nie, Z. Self-Assembly of Amphiphilic Plasmonic Micelle-like Nanoparticles in Selective Solvents. J. Am. Chem. Soc. 2013, 135, 7974-7984.

(26) Sánchez-Iglesias, A.; Grzelczak, M.; Altantzis, T.; Goris, B.; et al. Hydrophobic Interactions Modulate Self-Assembly of Nanoparticles. ACS Nano 2012, 6, 11059-11065.

(27) Maiolo, D.; Del Pino, P.; Metrangolo, P.; Parak, W. J.; Baldelli Bombelli, F. Nanomedicine Delivery: Does Protein Corona Route to the Target or off Road? Nanomedicine (London, U. K.) 2015, 10, $3231-3247$

(28) Wösten, H. A. B.; de Vocht, M. L. Hydrophobins, the Fungal Coat Unravelled. Biochim. Biophys. Acta, Rev. Biomembr. 2000, 1469, $79-86$.

(29) Milani, R.; Monogioudi, E.; Baldrighi, M.; Cavallo, G.; Arima, V.; Marra, L.; Zizzari, A.; Rinaldi, R.; Linder, M.; Resnati, G.; Metrangolo, P. Hydrophobin: Fluorosurfactant-like Properties without Fluorine. Soft Matter 2013, 9, 6505.

(30) Taniguchi, S.; Sandiford, L.; Cooper, M.; Rosca, E. V.; Ahmad Khanbeigi, R.; Fairclough, S. M.; Thanou, M.; Dailey, L. A.; Wohlleben, W.; von Vacano, B.; T. M. de Rosales, R.; Dobson, P. J.; Owen, D. M.; Green, M. Hydrophobin-Encapsulated Quantum Dots. ACS Appl. Mater. Interfaces 2016, 8, 4887-4893.

(31) Sarparanta, M.; Bimbo, L. M.; Rytkönen, J.; Mäkilä, E.; Laaksonen, T. J.; Laaksonen, P.; Nyman, M.; Salonen, J.; Linder, M. B.; Hirvonen, J.; Santos, H. A.; Airaksinen, A. J. Intravenous Delivery of Hydrophobin-Functionalized Porous Silicon Nanoparticles: Stability, Plasma Protein Adsorption and Biodistribution. Mol. Pharmaceutics 2012, 9, 654-663.

(32) Aimanianda, V.; Bayry, J.; Bozza, S.; Kniemeyer, O.; Perruccio, K.; Elluru, S. R.; Clavaud, C.; Paris, S.; Brakhage, A. A.; Kaveri, S. V.; Romani, L.; Latgé, J.-P. Surface Hydrophobin Prevents Immune Recognition of Airborne Fungal Spores. Nature 2009, 460, 11171121. 
(33) Pigliacelli, C.; D’Elicio, A.; Milani, R.; Terraneo, G.; Resnati, G.; Baldelli Bombelli, F.; Metrangolo, P. Hydrophobin-Stabilized Dispersions of PVDF Nanoparticles in Water. J. Fluorine Chem. 2015, 177, $62-69$.

(34) Gazzera, L.; Milani, R.; Pirrie, L.; Schmutz, M.; Blank, C.; Resnati, G.; Metrangolo, P.; Krafft, M. P. Design of Highly Stable Echogenic Microbubbles Through Controlled Assembly of Their Hydrophobin Shell. Angew. Chem., Int. Ed. 2016, 55, 10263-10267.

(35) Laaksonen, P.; Kainlauri, M.; Laaksonen, T.; Shchepetov, A.; Jiang, H.; Ahopelto, J.; Linder, M. B. Interfacial Engineering by Proteins: Exfoliation and Functionalization of Graphene by Hydrophobins. Angew. Chem., Int. Ed. 2010, 49, 4946-4949.

(36) Gazzera, L.; Corti, C.; Pirrie, L.; Paananen, A.; Monfredini, A.; Cavallo, G.; Bettini, S.; Giancane, G.; Valli, L.; Linder, M. B.; Resnati, G.; Milani, R.; Metrnagolo, P. Hydrophobin as a Nanolayer Primer That Enables the Fluorinated Coating of Poorly Reactive Polymer Surfaces. Adv. Mater. Interfaces 2015, 2, 1500170.

(37) Raffaini, G.; Milani, R.; Ganazzoli, F.; Resnati, G.; Metrangolo, P. Atomistic Simulation of Hydrophobin \{HFBII Conformation in Aqueous and Fluorous Media and at the Water/vacuum Interface. J. Mol. Graphics Modell. 2016, 63, 8-14.

(38) Bosetti, R. Cost-Effectiveness of Nanomedicine: The Path to a Future Successful and Dominant Market? Nanomedicine (London, U. K.) 2015, 10, 1851-1853.

(39) Reineck, P.; Gómez, D.; Ng, S. H.; Karg, M.; Bell, T.; Mulvaney, P.; Bach, U. Distance and Wavelength Dependent Quenching of Molecular Fluorescence by Au@SiO2 Core-Shell Nanoparticles. ACS Nano 2013, 7, 6636-6648.

(40) Shen, G.; Xing, R.; Zhang, N.; Chen, C.; Ma, G.; Yan, X. Interfacial Cohesion and Assembly of Bioadhesive Molecules for Design of Long-Term Stable Hydrophobic Nanodrugs toward Effective Anticancer Therapy. ACS Nano 2016, 10, 5720-5729.

(41) Grassi, M.; Grassi, G.; Lapasin, R.; Colombo, I. Understanding Drug Release and Adsorption Mechanism; CRC Press: New York, 2006.

(42) Chen, H.; Kim, S.; He, W.; Wang, H.; Low, P. S.; Park, K.; Cheng, J. X. Fast Release of Lipophilic Agents from Circulating PEGPDLLA Micelles Revealed by in Vivo Forster Resonance Energy Transfer Imaging. Langmuir 2008, 24, 5213-5217.

(43) Zou, P.; Chen, H.; Paholak, H. J.; Sun, D. Noninvasive Fluorescence Resonance Energy Transfer Imaging of in Vivo Premature Drug Release from Polymeric Nanoparticles. Mol. Pharmaceutics 2013, 10, 4185-4194.

(44) Pezzoli, D.; Zanda, M.; Chiesa, R.; Candiani, G. The Yin of Exofacial Protein Sulfhydryls and the Yang of Intracellular Glutathione in in Vitro Transfection with SS14 Bioreducible Lipoplexes. J. Controlled Release 2013, 165, 44-53.

(45) Prasetyanto, E. A.; Bertucci, A.; Septiadi, D.; Corradini, R; Castro-Hartmann, P.; De Cola, L. Breakable Hybrid Organosilica Nanocapsules for Protein Delivery. Angew. Chem., Int. Ed. 2016, 55, 3323-3327.

(46) Tanei, T.; Leonard, F.; Liu, X.; Alexander, J. F.; Saito, Y.; et al. Redirecting Transport of Nanoparticle Albumin-Bound Paclitaxel to Macrophages Enhances Therapeutic Efficacy against Liver Metastases. Cancer Res. 2016, 76, 429-439.

(47) Xu, R.; Zhang, G.; Mai, J.; Deng, X.; Segura-Ibarra, V.; Wu, S.; Shen, J.; Liu, H.; Hu, Z.; Chen, L.; Huang, Y.; Koay, E.; Huang, Y.; Liu, J.; Ensor, J. E.; Blanco, E.; Liu, X.; Ferrari, M.; Shen, H. An Injectable Nanoparticle Generator Enhances Delivery of Cancer Therapeutics. Nat. Biotechnol. 2016, 34, 414-418.

(48) Wang, Y.; Liu, D.; Zheng, Q.; Zhao, Q.; Zhang, H.; Ma, Y.; Fallon, J. K.; Fu, Q.; Haynes, M. T.; Lin, G.; Zhang, R.; Wang, D.; Yang, X.; Zhao, L.; He, Z.; Liu, F. Disulfide Bond Bridge Insertion Turns Hydrophobic Anticancer Prodrugs into Self-Assembled Nanomedicines. Nano Lett. 2014, 14, 5577-5583.

(49) Yang, X.; Yang, M.; Pang, B.; Vara, M.; Xia, Y. Gold Nanomaterials at Work in Biomedicine. Chem. Rev. 2015, 115, 10410-10488
(50) Saha, K.; Agasti, S. S.; Kim, C.; Li, X.; Rotello, V. M. Gold Nanoparticles in Chemical and Biological Sensing. Chem. Rev. 2012, 112, 2739-2779.

(51) Pang, B.; Zhao, Y.; Luehmann, H.; Yang, X.; Detering, L.; You, M.; Zhang, C.; Zhang, L.; Li, Z.-Y.; Ren, Q.; Liu, Y.; Xia, Y. 64 CuDoped PdCu@Au Tripods: A Multifunctional Nanomaterial for Positron Emission Tomography and Image-Guided Photothermal Cancer Treatment. ACS Nano 2016, 10, 3121-3131.

(52) Hembury, M.; Chiappini, C.; Bertazzo, S.; Kalber, T. L.; Drisko, G. L.; Ogunlade, O.; Walker-Samuel, S.; Krishna, K. S.; Jumeaux, C.; Beard, P.; Kumar, C. S. S. R.; Porter, A. E.; Lythgoe, M. F.; Boissière, C.; Sanchez, C.; Stevens, M. M. Gold-Silica Quantum Rattles for Multimodal Imaging and Therapy. Proc. Natl. Acad. Sci. U. S. A. 2015, 112, 1959-1964.

(53) Soikkeli, M.; Kurppa, K.; Kainlauri, M.; Arpiainen, S.; Paananen, A.; Gunnarsson, D.; Joensuu, J. J.; Laaksonen, P.; Prunnila, M.; Linder, M. B.; Ahopelto, J. Graphene Biosensor Programming with Genetically Engineered Fusion Protein Monolayers. ACS Appl. Mater. Interfaces 2016, 8, 8257-8264.

(54) Askolin, S.; Nakari-Setälä, T.; Tenkanen, M. Overproduction, Purification, and Characterization of the Trichoderma Reesei Hydrophobin HFBI. Appl. Microbiol. Biotechnol. 2001, 57, 124-130.

(55) Bigini, P.; Previdi, S.; Casarin, E.; Silvestri, D.; Violatto, M. B.; Facchin, S.; Sitia, L.; Rosato, A.; Zuccolotto, G.; Realdon, N.; Fiordaliso, F.; Salmona, M.; Morpurgo, M. Vivo Fate of Avidin-Nucleic Acid Nanoassemblies as Multifunctional Diagnostic Tools. ACS Nano 2014, 8, 175-187.

(56) Fruscio, R.; Lissoni, A. A.; Frapolli, R.; Corso, S.; Mangioni, C.; D'Incalci, M.; Zucchetti, M. Clindamycin-paclitaxel Pharmacokinetic Interaction in Ovarian Cancer Patients. Cancer Chemother. Pharmacol. 2006, 58, 319-325.

(57) Recordati, C.; De Maglie, M.; Bianchessi, S.; Argentiere, S.; Cella, C.; Mattiello, S.; Cubadda, F.; Aureli, F.; D’Amato, M.; Raggi, A.; Lenardi, C.; Milani, P.; Scanziani, E. Tissue Distribution and Acute Toxicity of Silver after Single Intravenous Administration in Mice: Nano-Specific and Size-Dependent Effects. Part. Fibre Toxicol. 2015, 13,12 . 